\title{
Leptina, Ghrelina e Exercício Físico
}

\begin{abstract}
RESUMO
A obesidade é atualmente um problema de saúde pública que provoca sérias conseqüências sociais, físicas e psicológicas. A etiologia da obesidade não é de fácil identificação, uma vez que a mesma é caracterizada como doença multifatorial de complexa interação entre fatores comportamentais, culturais, genéticos, fisiológicos e psicológicos. Recentes avanços na área de endocrinologia e metabolismo mostram que, diferentemente do que se acreditava há alguns anos, o adipócito sintetiza e libera diversas substâncias, não sendo apenas uma célula armazenadora de energia. Entre as substâncias liberadas pelo adipócito incluem-se a adiponectina, o fator de necrose tumoral- $\alpha$, a interleucina- 6 e a leptina. Especificamente, a leptina desempenha importante papel no controle da ingestão alimentar e no controle do peso corporal em mamíferos. Além disso, o hormônio ghrelina, recentemente descoberto, também parece influenciar o metabolismo energético e a obesidade. As alterações que o exercício físico provoca na fisiologia endócrino-metabólica podem contribuir sobremaneira para a prática clínica. Assim, essa revisão abordará os conhecimentos mais recentes sobre a leptina, a ghrelina e o papel dos diferentes tipos de exercício físico sobre estes hormônios. Os trabalhos mostram que a relação entre o exercício físico e a concentração plasmática desses peptídeos ainda não está clara. As razões para isso poderiam ser devidas aos diferentes protocolos de treinamento físico empregados nos estudos. Além disso, diferenças genéticas também podem explicar as discrepâncias entre os resultados obtidos em seres humanos, pois a existência de polimorfismo em alguns genes pode acarretar respostas celulares diferentes frente ao exercício físico. (Arq Bras Endocrinol Metab 2007;51/1:25-33)
\end{abstract}

Descritores: Exercício físico; Leptina; Ghrelina; Obesidade

\section{ABSTRACT}

\section{Leptin, Ghrelin, and Physical Exercise.}

Obesity is a major public health problem in the Western world resulting in serious social, physical and psychological damages. The genesis of obesity is complex involving a variety of factors such as genetic, psychological, metabolic and environmental factors. Progress in endocrinology and metabolism show that adipocyte is considered now as an endocrine tissue producing several substance including adiponectin, tumor necrosis factor- $\alpha$, interleukin- 6 and leptin. Specifically, leptin is the main peptide produced by the adipocyte and its serum concentration represents an important peripheral signal in the regulation of food intake and energy expenditure in mammals. In addition to leptin, a new peptide was discovered recently named ghrelin. Ghrelin, a peptide hormone identified in the stomach, is directly involved with the regulation of energy balance and obesity. Physical exercise has been used as a non-pharmacological tool in management of body weight and the effect of physical activity on weight control is an important issue for clinical studies in endocrinology field. Thus, this review will attempt to update the knowledge of leptin and ghrelin on the body weight regulation and the effect of exercise training on these peptide concentrations. It can be concluded that the relationship between physical exercise and the plasma concentration of these peptides is not clear. The reasons for that could be related to the differences in duration, intensity and frequency of the training program employed in each study. Indeed, most of the studies have not analyzed the intensity of training program by either plasma lactate concentration or maximum oxygen consumption. On the other hand, genetic basis could also explain the discrepancies found in some studies, since it has been shown that polymorphism for a variety of genes might be an important factor to determine the differences of cellular response to physical training. (Arq Bras Endocrinol Metab 2007;51/1:25-33)

Keywords: Physical exercise; Leptin; Ghrelin; Obesity

\section{revisão}

\author{
GustaVo R. DA MOtA \\ ANGELINA ZANESCO
}

Departamento de Educação

Física, Instituto de Biociências, Universidade Estadual Paulista

(UNESP), Rio Claro, SP.
Recebido em 05/10/05

Revisado em 30/03/06

Aceito em 10/07/06 
A COMPREENSÃO DOS FATORES que influenciam o equilíbrio energético e a manutenção do peso corporal é de grande relevância nos dias atuais, uma vez que o número de pessoas com sobrepeso e obesidade tem aumentado de forma crescente no Brasil e em diversas partes do mundo. A obesidade é atualmente um problema de saúde pública que provoca sérias conseqüências sociais, físicas e psicológicas. As conseqüências físicas estão diretamente associadas a maiores riscos de morbidade e mortalidade, bem como doenças crônicas como hipertensão arterial, diabetes mellitus tipo 2 e dislipidemias (1). Deve-se salientar que a etiologia da obesidade não é de fácil identificação, uma vez que a mesma é caracterizada como doença multifatorial de complexa interação entre fatores comportamentais, culturais, genéticos, fisiológicos e psicológicos (2).

Recentes avanços na área de endocrinologia e metabolismo mostram que, diferentemente do que se acreditava há alguns anos, o adipócito não é apenas uma célula armazenadora de energia, mas sim capaz de sintetizar e liberar diversas substâncias, sendo hoje considerado um órgão endócrino (3). Entre as substâncias liberadas pelo adipócito incluem-se a adiponectina, o fator de necrose tumoral alfa (TNF- $\alpha$ ), alguns hormônios sexuais, a interleucina-6 e a leptina. Especificamente, a leptina desempenha importante papel no controle da ingestão alimentar e no controle do peso corporal em mamíferos (4). Além dos avanços no estudo da célula adiposa, um novo hormônio relacionado ao metabolismo foi descoberto recentemente, a ghrelina. A ghrelina é um peptídeo produzido pelas células do estômago e está diretamente envolvida na regulação do balanço energético em curto prazo. A atividade física representa a variável mais flexível do gasto energético em humanos (5). Considerando o papel da leptina e da ghrelina no gasto energético e a influência da atividade física na manutenção da perda de peso $(6,7)$, essa revisão abordará os conhecimentos existentes sobre esses dois hormônios e a influência que o exercício físico pode promover no controle do peso corporal e nos níveis desses peptídeos.

\section{LEPTINA}

O hormônio denominado leptina foi descoberto no final de 1994 e rapidamente ganhou grande destaque na literatura científica internacional (8). Desde então, um grande número de pesquisas foi desenvolvido sobre o tema para melhor compreensão de sua função, principalmente com relação à regulação da ingestão energética e, conseqüentemente, o papel que este hormônio desempenha na obesidade (9-11).

O nome leptina é derivado do grego leptos, que significa magro $(12,13)$. A leptina é um hormônio peptídico formado por 167 aminoácidos, transcrito a partir do gene ob, que foi originalmente clonado em camundongos. A mutação desse gene, ou sua deficiência, acarreta obesidade severa e diabetes tipo II nesses animais. O gene da leptina humana está localizado no cromossomo 7q31 e seu DNA tem mais de 15.000 pares de bases e existem três exons (8). A leptina é produzida, principalmente, no tecido adiposo branco e, quando injetada em camundongo ob/ob, o qual tem deficiência genética desse peptídeo, reduz o consumo de alimentos e aumenta o gasto energético (14). Por outro lado, quando a leptina é injetada no camundongo $\mathrm{db} / \mathrm{db}$, que apresenta deficiência do receptor de leptina, não há nenhuma perda de peso corporal ou diminuição do consumo energético (12).

Em humanos, a concentração plasmática de leptina está parcialmente relacionada ao tamanho da massa de tecido adiposo presente no corpo $(15,16)$, pois seus níveis plasmáticos diferem em indivíduos com mesmo índice de massa corporal (17). Além disso, mulheres geralmente apresentam níveis maiores de leptina do que homens (11), e em algumas patologias, como o diabetes mellitus do tipo II, os níveis plasmáticos de leptina apresentam-se elevados. Assim, homens não diabéticos apresentam valores médios de, aproximadamente, $10 \mathrm{ng} / \mathrm{ml}$ comparados com 20 $\mathrm{ng} / \mathrm{ml}$ em homens diabéticos. Esses valores são maiores em mulheres diabéticas $(55 \mathrm{ng} / \mathrm{ml})$ comparadas a mulheres não diabéticas $(10 \mathrm{ng} / \mathrm{ml})(18)$.

A tabela 1 apresenta valores de leptina plasmática encontrados em alguns estudos, com diferentes populações.

A estrutura da leptina é semelhante à das citocinas, como a interleucina-2. Uma de suas funções está relacionada ao controle da ingestão alimentar, atuando em células do núcleo arqueado do hipotálamo, no sistema nervoso central $(3,10)$.

Além dos adipócitos, ela é sintetizada no estômago (19), na placenta (20) e na glândula mamária (21). Outro aspecto interessante com relação à leptina é que sua produção difere entre os adipócitos. Sabe-se, por exemplo, que a produção de leptina é maior no tecido adiposo subcutâneo do que no tecido visceral $(11,22)$.

Os níveis de leptina podem ser influenciados por diversos fatores e substâncias. Uma exposição prolongada ao jejum diminui os níveis plasmáticos de leptina, ao passo que alimentação excessiva aumenta sua concentração (23). A composição da dieta, em 
Tabela 1. Valores basais de concentração plasmática de leptina em diferentes populações.

\begin{tabular}{lccccc}
\hline GRUPOS & $\mathbf{N}$ & Idade (anos) & IMC (kg/m $\left.{ }^{2}\right)$ & Leptina (ng/ml) & Referências \\
Homens & 51 & $24 \pm 6$ & $25,5 \pm 5,0$ & $4,6 \pm 4,4$ & 33 \\
Mulheres & 46 & $24 \pm 6$ & $23,0 \pm 3,5$ & $11,9 \pm 8,5$ & 33 \\
Homens & 281 & $50 \pm 16$ & $31,0 \pm 7,9$ & $12,7 \pm 13,0$ & 16 \\
Mulheres & 687 & $46 \pm 14$ & $37,5 \pm 11,4$ & $42,7 \pm 29,1$ & 16 \\
Ultramaratonistas (Homens) & 14 & $41 \pm 13$ & $23,9 \pm 2,0$ & $2,64 \pm 0,94$ & 53 \\
Homens treinados em endurance & 20 & $21 \pm 4$ & $21,6 \pm 2,0$ & $2,05 \pm 0,7$ & 34 \\
Homens treinados em exercícios com pesos & 17 & $23 \pm 2$ & $23,6 \pm 1,4$ & $2,4 \pm 0,86$ & 34 \\
Pacientes com deficiência de GH (mulheres) & 15 & $47 \pm 7$ & $25 \pm 3$ & $21,5 \pm 8$ & 35 \\
\hline Pacientes com deficiência de GH (homens) & 21 & $45 \pm 7$ & $26 \pm 3$ & $5,2 \pm 2,2$ & 35 \\
\hline
\end{tabular}

Os valores são expressos em média \pm desvio-padrão.

especial o consumo de macro-nutrientes (24) e micronutrientes, tais como o zinco $(25)$, e fatores hormonais influenciam seus níveis (26). Estudo avaliando adipócitos humanos isolados mostra que a infusão prolongada ou doses supra-fisiológicas de insulina aumentam os níveis circulantes de leptina (26), assim como a administração exógena de glicocorticóides (27). Por outro lado, isoproterenol e agonistas dos adrenorreceptores $\beta_{3}$ reduzem a expressão de mRNA e os níveis plasmáticos de leptina, bem como o fumo de cigarros (28-30). Várias citocinas, tais como o TNF $\alpha$ (31), interleucina-l e interleucina- 6 , também aumentam a expressão de RNAm para a síntese de leptina (32).

\section{Mecanismos de ação da leptina na ingestão alimentar}

A leptina reduz o apetite através da inibição da liberação de neuropeptídeos relacionados ao apetite, como o neuropeptídeo Y (NPY) e o peptídeo relacionado à proteína Agouti (AGRP), que são peptídeos orexígenos (10). Atua também através do aumento da expressão e liberação de neuropeptídeos anorexígenos, como o hormônio estimulante de melanócito $(\alpha-$ MSH), o hormônio liberador de corticotropina $(\mathrm{CRH})$ e substâncias sintetizadas em resposta à anfetamina e cocaína, no sistema nervoso central. Assim, altos níveis de leptina reduzem a ingestão alimentar enquanto baixos níveis induzem hiperfagia. Isso é comprovado em animais de laboratório obesos que apresentam baixos níveis ou total deficiência de leptina. Por isso, esse hormônio ficou conhecido como o "hormônio da saciedade". Uma falha na sua produção e/ou na sua ação sobre os receptores hipotalâmicos poderia desequilibrar positivamente $\mathrm{O}$ balanço energético, gerando o quadro de obesidade
(12). No entanto, estudos realizados em humanos mostram que os níveis plasmáticos de leptina são elevados em obesos quando comparados com seus congêneres magros (16,33-35). Crianças pré-púberes e adolescentes obesos também apresentam níveis de leptina mais elevados do que indivíduos magros da mesma faixa etária (36).

Além de seu importante papel no metabolismo, a leptina também parece participar no controle dos sistemas hematopoiético (37), imune (38), reprodutor (39), cardiovascular $(40,41)$ e no metabolismo ósseo (42).

\section{Resistência à leptina na obesidade humana} Quando os primeiros trabalhos reportaram que a administração exógena de leptina em camundongos $o b / o b$ (obesos) provocava redução da hiperfagia e do peso corporal nesses animais (14), gerou-se grande expectativa a respeito de sua utilidade no tratamento da obesidade em seres humanos. A esperança de utilizar a leptina exógena no tratamento de humanos obesos foi ainda intensificada pois, até o momento, existem apenas duas drogas aprovadas pelo Food and Drug Administration para o tratamento e controle da obesidade, a sibutramina e o orlistat (43). Assim, a utilização de leptina exógena poderia ser uma nova abordagem no tratamento da obesidade, pois estudos mostraram que a administração de leptina em roedores promoveu redução do peso corporal $(10,14)$. A deficiência de leptina em obesos que respondem a sua administração exógena é baixa, cerca de 5 a $10 \%$. Realmente, poucos casos em humanos foram caracterizados clinicamente como apresentando deficiência de leptina. Além disso, a administração exógena de leptina, como tratamento da obesidade humana, não reproduziu os resultados obtidos em animais de 
laboratório (44). A grande maioria de pessoas obesas apresenta quadro de resistência à leptina, ou seja, apresentam grandes quantidades de leptina na circulação, mas seu efeito de saciedade e inibição do apetite não ocorre. Dessa forma, a eficácia da administração de leptina a esses indivíduos permanece duvidosa e sua eficácia ainda não foi comprovada. Seria interessante identificar quais os possíveis defeitos nos receptores e mecanismos pós-receptor de leptina, no hipotálamo, que poderiam ser responsáveis pela resistência à leptina, encontrada em humanos obesos $(12,45)$.

\section{Leptina e exercício físico em ratos}

Vários trabalhos têm mostrado que os níveis plasmáticos de leptina são reduzidos após o treinamento físico em animais (45-47). Steinberg e cols. (45) constataram que o treinamento de endurance em esteira, com velocidade de $21 \mathrm{~m} / \mathrm{min}, 5$ dias por semana, 2 horas/sessão, durante 4 semanas reverte, parcialmente, a resistência à leptina induzida pela dieta hiperlipídica. Resultados semelhantes foram encontrados por Jen e cols. (47) e Estadella e cols. (48), os quais verificaram que o treinamento de endurance em ratos evitava o aumento dos níveis plasmáticos de leptina induzido por dieta hiperlipídica. Estadella e cols. (48) observaram que a diminuição nos níveis de leptina era acompanhada, também, por menores ganhos de peso corporal e massa adiposa, e níveis mais baixos de triglicérides séricos e insulina. Por outro lado, no estudo de Jen e cols. (47) a queda nos níveis de leptina foi independente da redução na gordura corporal. Portanto, os estudos em animais mostram, de maneira geral, que os níveis plasmáticos de leptina são reduzidos após um programa de treinamento físico.

\section{Leptina e exercícios aeróbios em humanos}

Nos últimos anos, foram conduzidos diversos estudos, inclusive de revisão, com o intuito de verificar a relação existente entre o tipo de atividade física praticado, a composição corporal e os níveis plasmáticos de leptina em atletas, pessoas ativas e sedentárias $(33,34,49-52)$.

Com relação ao exercício físico dinâmico de longa duração, com predominância aeróbia, os dados são conflitantes. Alguns autores não observaram qualquer alteração nos níveis plasmáticos de leptina $(33,49)$, enquanto outros mostraram ter havido redução nos mesmos $(51,53)$.

\section{Leptina e exercício agudo}

Diversos estudos mostram que os níveis plasmáticos de leptina não se alteram em função de exercícios aeróbios agudos em atletas ou não atletas (54-57).
Weltman e cols. (55) desenvolveram um protocolo de exercício com o objetivo de verificar se a concentração plasmática de leptina era dependente da intensidade do exercício aeróbio (corrida em esteira rolante por 30 minutos). Para tanto, os voluntários executaram o mesmo exercício, em diferentes dias, com diferentes intensidades baseadas no limiar de lactato sangǘńneo individual. Os resultados demonstraram que a intensidade do exercício aeróbio não modifica os níveis plasmáticos de leptina logo após o esforço, nem após a recuperação. Mais recentemente, Zoladz e cols. (57) verificaram que os níveis de leptina permanecem inalterados após uma sessão aguda de exercício aeróbio, confirmando resultados prévios.

Dirlewanger e cols. (58), estudando os efeitos da atividade física moderada sobre a concentração plasmática de leptina, estudaram 11 indivíduos magros e saudáveis em três ocasiões diferentes, durante 3 dias consecutivos: (a) em equilíbrio energético sem exercício; (b) em estado de equilíbrio energético negativo, utilizando o exercício de pedalar duas vezes por dia, por 30 minutos, com $60 \mathrm{~W}$ de intensidade, e (c) em situação de equilíbrio energético com exercício. A concentração plasmática de leptina, em jejum no quarto dia de cada ocasião, não foi diferente para os três procedimentos experimentais. Com isso, os autores concluíram que o exercício físico não tem efeito direto sobre a leptinemia quando a composição corporal não é alterada.

Estudos em crianças e adolescentes sedentários, com e sem obesidade, mostraram que teste incremental, com aumento progressivo da intensidade do exercício, executado em esteira rolante não modificava a leptinemia (36).

A ausência de alterações na concentração de leptina pode estar relacionada ao tempo de coleta após a sessão de exercício físico. Como a leptina está, direta ou indiretamente, envolvida no controle do equilíbrio energético do organismo em longo prazo, poderia haver necessidade de um tempo maior para a coleta, para que modificações fossem realmente notadas em resposta ao exercício. Neste sentido, Landt e cols. (53) demonstraram que a concentração plasmática de leptina diminuía 32\% em ultramaratonistas após atividade extenuante constituída de 101 milhas de corrida, com $35,8 \pm 11,1$ horas de duração e grande gasto energético. Com relação ao tempo de coleta, Nindl e cols. (59) estudaram indivíduos fisicamente ativos empregando exercícios com pesos e observaram que, somente após 9 horas, os níveis de leptina estavam diminuídos. De forma semelhante, outro estudo mostrou que a concentração plasmática de leptina só 
diminuía após 24 e 48 horas depois da interrupção da sessão de exercícios. Nesse caso, o esforço era de natureza aeróbia, a $70 \%$ do $\mathrm{VO}_{2}$ máx, durante uma hora (60). Resultados parecidos também foram relatados por Keller e cols. (61), que verificaram que o ato de pedalar por três horas diminuiu a concentração plasmática de leptina.

\section{Leptina e exercício crônico}

Em geral, em estudos crônicos de curta duração, não superior a 12 semanas, observa-se redução da leptinemia tanto em humanos (62) quanto em animais de laboratório (45-48). Por outro lado, existem resultados conflitantes quando se analisa o estudo do efeito do exercício físico de longa duração sobre os níveis circulantes de leptina. Alguns estudos mostram diminuição $(63,64)$ e outros nenhuma alteração $(33,51,65)$.

Pérusse e cols. (33) recrutaram 97 indivíduos adultos obesos e sedentários, sendo 51 homens e 46 mulheres, que treinaram por 20 semanas, em cicloergômetro, com freqüência semanal de 3 vezes e intensidade inicial de $55 \%$ e final de $75 \%$ do $\mathrm{VO}_{2}$ máx. Os resultados mostraram que, apesar de o $\mathrm{VO}_{2}$ máx ter melhorado significativamente após as 20 semanas, tanto homens quanto mulheres não apresentaram qualquer modificação nos níveis plasmáticos de leptina. Recentemente, em nosso laboratório avaliamos o efeito do exercício aeróbio por 24 semanas em mulheres obesas de meia-idade, a $50 \%$ da freqüência cardíaca máxima, e também não encontramos qualquer alteração nos níveis séricos de leptina (65).

Por outro lado, Pasman e cols. (63), investigando homens obesos durante 16 meses de intervenção dietética e de exercícios aeróbios, verificaram que os níveis plasmáticos de leptina eram diminuídos no grupo treinado fisicamente. Similarmente, após um ano de intervenção com exercícios aeróbios e diminuição de consumo alimentar, foi observada redução na concentração de leptina em homens com síndrome metabólica (64).

Em atletas, poucos estudos foram realizados na tentativa de encontrar alguma correlação entre os níveis plasmáticos de leptina e alguma variável que poderia influenciar a performance dos mesmos. Uma pesquisa recente mostrou que a concentração plasmática de leptina não estava alterada neste tipo particular de população. Tanto em atletas que praticam exercícios de natureza aeróbia (51) como naqueles que executam altos níveis de força muscular (34) os níveis plasmáticos de leptina são semelhantes ao de seus congêneres não-atletas.
Portanto, até o momento não existem resultados conclusivos sobre os efeitos do exercício aeróbio sobre os níveis plasmáticos de leptina em humanos. A razão para isso poderia estar relacionada ao tempo de coleta após a sessão do exercício, assim coleta de sangue por 24 horas seria necessária para monitorar a evolução temporal da concentração plasmática da leptina em resposta ao exercício. Bases genéticas também poderiam contribuir para os resultados conflitantes. Sabe-se que a existência de polimorfismo em alguns genes pode influenciar a resposta do organismo frente ao exercício físico. No entanto, pouco se sabe sobre a relação entre genética e exercício físico, até o momento.

\section{Leptina e exercícios com pesos em humanos}

Como em quase todas outras áreas do conhecimento científico relacionado ao movimento humano, a maioria dos estudos sobre leptina estudou o efeito do treinamento aeróbio, e poucos trabalhos existem relacionados ao treinamento com pesos (musculação) e leptina. Um estudo envolvendo jovens fisiculturistas e jovens sedentários, com sobrepeso ou com peso considerado normal, demonstrou que os níveis de leptina eram semelhantes entre os grupos e que o treinamento com pesos com objetivo de hipertrofia muscular, portanto, não influenciava a concentração de leptina independentemente das variações na composição corporal encontradas (66).

Kanaley e cols. (18), estudando pacientes diabéticos, observaram redução no nível plasmático de leptina na fase aguda do programa de treinamento físico com pesos, mas nenhuma alteração foi encontrada, cronicamente, após seis semanas desse tipo de treinamento. Outro estudo interessante, com mulheres obesas após a menopausa, encontrou redução de $36 \%$ no nível plasmático de leptina após 16 semanas de treinamento com pesos associado à redução de peso corporal. Por outro lado, as mulheres do mesmo estudo que faziam apenas o treinamento com pesos, mas não participavam do esquema de redução ponderal, não apresentavam qualquer alteração no nível plasmático de leptina (67).

Recente estudo comparou diferentes protocolos comumente utilizados de treinamento com pesos (força máxima, hipertrofia muscular e resistência muscular localizada), em dias diferentes, além de outro dia de controle, sem exercício algum, nas mesmas condições, para observar o comportamento dos níveis plasmáticos de leptina. Não houve nenhuma alteração na concentração plasmática de leptina entre os diferentes tipos de protocolos empregados (68). 
Assim, estes estudos mostram que os níveis de leptina parecem não ser influenciados pelos exercícios com pesos, independentemente do protocolo empregado, seja realizado baixo número de repetições com grande peso ou alto número de repetições empregadas com pequeno peso opondo-se ao movimento.

\section{GHRELINA}

A ghrelina é um hormônio relativamente novo que foi descoberto em 1999 por um grupo de pesquisadores japoneses. É um peptídeo composto por 28 aminoácidos que apresenta duas isoformas: a acilada e a não acilada (69). Secretada principalmente pelas células do estômago, a ghrelina atua no sistema nervoso central sinalizando a necessidade de ingerir alimentos. Seu papel no controle do metabolismo tem sido recentemente estudado, e observou-se que a administração de ghrelina em ratos induz ao comportamento de ingestão alimentar, redução no gasto metabólico e obesidade (70). A ghrelina é sintetizada pelas células da camada mucosa da região fúndica do estômago. Além das células do estômago, uma proporção menor de ghrelina é sintetizada no hipotálamo, duodeno, coração, rins e nos pulmões (71,72). Assim, a secreção de ghrelina é inibida pela ingestão de nutrientes, pois estes estimulam a secreção de vários outros hormônios intestinais e pancreáticos que controlariam a sua liberação (73). O núcleo arqueado do hipotálamo é o maior sítio regulador da ingestão alimentar e do peso corporal, contendo neurônios orexígenos e anorexígenos. Os neurônios orexígenos liberam o NPY e o AGRP. A ghrelina estimula a liberação destes peptídeos que são potentes estimuladores do apetite (69).

Os níveis plasmáticos de ghrelina são baixos em sujeitos obesos quando comparados com sujeitos magros (74), enquanto indivíduos com dieta de baixo teor calórico, sob treinamento físico crônico, ou com anorexia nervosa apresentam níveis de ghrelina elevados. As causas destas diferenças ainda não são conhecidas. Além disso, estudos recentes observaram que os níveis séricos de ghrelina são três a quatro vezes maiores em crianças com a Síndrome de Prader-Willi do que obesos controles. Especula-se que a ghrelina pode ser um fator orexígeno que contribui acentuadamente para o apetite voraz e, conseqüentemente, a obesidade vista nessa síndrome (76).

O nome ghrelina vem do prefixo ghre de origem Proto-Indo-Européia, que dá origem a growth, do inglês "crescimento" (69). O sufixo relin (em inglês release) dá um sentido semântico (liberador de $\mathrm{GH})$. A ghrelina é um poderoso estimulador da liberação do hormônio de crescimento $(\mathrm{GH})$, agindo diretamente nos somatotrófos hipofisários e indiretamente nos neurônios secretores de GHRH do núcleo arqueado do hipotálamo, através da ativação dos receptores GHS-Rla, pertencentes à família dos receptores acoplados à proteína G. Assim, a grelina é o ligante endógeno dos receptores órfãos GHS que estão presentes na hipófise e no hipotálamo (75). O papel da ghrelina no metabolismo de indivíduos normais foi estudado por diferentes laboratórios de pesquisa, mostrando que a ghrelina aumenta a ingestão alimentar e que, na situação pós-prandial, seus níveis ficam reduzidos. No entanto, em sujeitos obesos os níveis de ghrelina são baixos e sua concentração pós-prandial não se modifica em reposta à ingestão alimentar. As razões pelas quais os indivíduos obesos não apresentam mudança nos níveis plasmáticos de ghrelina após a ingestão alimentar ainda não são claras $(77,78)$.

Sabe-se que sujeitos obesos possuem hipossecreção de GH e ghrelina, e que a administração exógena de ghrelina nestes indivíduos não altera essa situação. Somente dieta hipocalórica, por longo período, e acentuada redução ponderal pode restaurar os níveis plasmáticos de ambos (79). Assim, é plausível admitir que outros fatores estão envolvidos na deficiência de $\mathrm{GH}$ e ghrelina em obesos.

\section{Ghrelina e exercício físico}

Poucos estudos foram realizados para verificar os efeitos do exercício físico sobre os níveis plasmáticos de ghrelina. Esse fato é compreensível em virtude de esse hormônio ser relativamente novo. Entretanto, os poucos trabalhos realizados mostram que os níveis plasmáticos de ghrelina não se modificam em resposta ao exercício. Um estudo foi realizado com o objetivo de caracterizar os níveis plasmáticos da ghrelina durante exercício submáximo, de caráter predominantemente aeróbio, em adultos saudáveis e deficientes de GH. Como era esperado, o exercício provocou aumento na concentração de $\mathrm{GH}$ ao final da sessão apenas nos indivíduos controle, enquanto que nos pacientes, deficientes de $\mathrm{GH}$, não houve qualquer alteração em relação aos valores de repouso. Interessantemente, os níveis plasmáticos de ghrelina também não se alteravam em quaisquer das situações, em ambos grupos (80). Analisando indivíduos obesos durante um programa de redução ponderal relativamente curto (três semanas), incluindo restrição calórica, exercícios físicos, aconselhamento psicológico e educação nutricional, observou-se que os obesos 
apresentavam menores níveis de ghrelina do que os indivíduos pertencentes ao grupo controle, de indivíduos saudáveis não obesos. Após o período de três semanas, apesar de os obesos reduzirem o peso corporal e o IMC, nenhuma alteração ocorreu com relação aos níveis de ghrelina $(81)$.

Foster-Schubert e cols. (82) verificaram que após um ano de intervenção com exercícios aeróbios em mulheres com sobrepeso, após a menopausa, a concentração plasmática de ghrelina aumentou. Esse aumento ocorreu mesmo na ausência de dieta ou alterações no consumo energético. Entretanto, segundo os autores, o incremento dos níveis de ghrelina ocorreu não pelo exercício em si, mas pela perda de peso (82). Cem dias de experimento com sessões de exercícios duas vezes ao dia em bicicleta ergométrica, com intenção de provocar equilíbrio energético negativo, não alterou os níveis plasmáticos de ghrelina em 12 pares de irmãos gêmeos (83).

Portanto, podemos concluir pelos estudos descritos acima que o exercício físico aeróbio não promove qualquer alteração nos níveis plasmáticos de ghrelina. A razão para isso ainda não é clara, pois ainda não se conhece qual ou quais seriam os mecanismos de controle neuro-hormonal da secreção de ghrelina.

\section{CONSIDERAÇÕES FINAIS}

O estudo do metabolismo e os fatores endógenos que contribuem para a manutenção do peso corporal são de grande relevância na área de endocrinologia e metabolismo. O papel do exercício físico na prevenção e/ou tratamento da obesidade é um tema atual e de fundamental importância para a manutenção da qualidade de vida da população. Assim, as possíveis alterações que o exercício físico provoca na fisiologia endócrino-metabólica podem contribuir sobremaneira para a prática clínica.

Em resumo, os trabalhos mostram que a relação entre o exercício físico e a concentração plasmática de leptina não está clara, alguns estudos mostram redução dos seus níveis enquanto outros falham em encontrar qualquer alteração. As razões para isso poderiam ser devidas aos diferentes protocolos de treinamento físico empregados nos estudos e ao tempo de coleta após a sessão do exercício físico. Com relação à ghrelina, os resultados mostram que seus níveis plasmáticos não se alteraram em resposta ao exercício físico. No entanto, ainda existem poucos trabalhos avaliando o efeito do exercício físico e os níveis de ghrelina. Além disso, diferenças genéticas devem ser levadas em consi- deração, uma vez que a existência de polimorfismo em alguns genes pode acarretar respostas celulares diferentes frente ao exercício físico.

\section{REFERÊNCIAS}

1. Bouchard C, Després JP. Variation in fat distribution with age and health implications. Am Acad Physical Educ 1989;22:78-106.

2. Bouchard C. Genetic factors in obesity. Med Clin North Am 1989;73(1):67-81.

3. Frühbeck G, Ambrosi JA, Muruzábal FJ, Burrell MA. The adipocyte: a model for integration of endocrine and metabolic signaling in energy metabolism regulation. Am J Physiol Endocrinol Metab 2001;280:E827-47.

4. Ahima RS, Flier JS. Leptin. Annu Rev Physiol 2000;62: 413-37.

5. Toth MJ, Poehlman ET. Effects of exercise on daily energy expenditure. Nutr Ver 1996;54:S140-8.

6. Votruba SB, Horvitz MA, Schoeller DA. The role of exercise in the treatment of obesity. Nutrition 2000;16:179-88.

7. American College of Sports Medicine. Position Stand on the Appropriate Intervention Strategies for Weight Regain for Adults. Med Sci Sports Exerc 2001;33(12):2145-56.

8. Zhang $Y$, Proença $R$, Maffei M, Barone M, Leopold L, Friedman JM. Positional cloning of the mouse obese gene and its human homologue. Nature 1994;372:425-32.

9. Mantzoros CS. The role of leptin in human obesity and disease: a review of current evidence. Ann Intern Med 1999;130:671-80.

10. Friedman JM. The function of leptin in nutrition, weight and physiology. Nutrition Rev 2002;60(10):S1-14.

11. Leibel RL. The role of leptin in the control of body weight. Nutrition Rev 2002;60(10):S15-9.

12. Bray G. Progress in understanding the genetics of obesity. $\mathbf{J}$ Nutr 1997;127:940S-42.

13. Auwerx J, Staels B. Leptin. Lancet 1998;351:737-42.

14. Mistry AM, Swick AG, Romsos DR. Leptin rapidly lowers food intake and elevates metabolic rates in lean and ob/ob mice. J Nutr 1997;127:2065-72.

15. Fors H, Matsuoka H, Bosaeus I, Rosberg S, Wikland KA, Bjarnason R. Serum leptin levels correlate with growth hormone secretion and body fat in children. $J$ Clin Endocrinol Metab 1999;84:3586-90.

16. Lee LH, Reed DR, Price RA. Leptin resistance is associated with extreme obesity and aggregates in families. Intern J Obes $2001 ; 25: 1471-3$.

17. Kennedy A, Gettys TW, Watson P, Wallace P, Ganaway E, Pan $Q$, et al. The metabolic significance of leptin in humans: gender-based differences in relationship to adiposity, insulin sensitivity, and energy expenditure. J Clin Endocrinol Metab 1997;82:1293-300.

18. Kanaley JA, Fenicchia LM, Miller CS, Ploutz-Synder LL, Weinstock RS, Carhart R, et al. Resting leptin responses to acute and chronic resistance training in type 2 diabetic men and women. Int $J$ Obes Relat Metab Disord 2001;25(10):1474-80.

19. Nishi Y, Isomoto H, Uotani S, Wen CY, Shikuwa S, Ohnita K, et al. Enhanced production of leptin in gastric fundic mucosa with Helicobacter pylori infection. World J Gastroenterol 2005;11(5):695-9.

20. Hassink SG, Lancey E, Sheslow DV, Smith-Kirwin SM, O'Connor DM, Considine RV, et al. Placental leptin: an important new growth factor in intrauterine and neonatal development? Pediatrics 1997;100(1):1-6.

21. Smith-Kirwin SM, O'Connor DM, De Johnston J, Lancey ED, Hassink SG, Funanage VL. Leptin expression in human mammary epithelial cells and breast milk. J Clin Endocrinol Metab 1998;83(5):1810-3. 
22. Considine RV, Cooksey RC, Williams LB, Fawcett RL, Zhang P, Ambrosius WT, et al. Hexosamines regulate leptin production in human subcutaneous adipocytes. J Clin Endocrinol Metab 2000;85:3551-6.

23. Kolaczynski JW, Considine RV, Ohannesian J, Marco C, Opentanova I, Nyce MR. Responses of leptin to short-term fasting and refeeding in humans. Diabetes 1996;45:1511-5.

24. Jenkins AB, Markovic TP, Fleury A, Campbell LV. Carbohydrate intake and short-term regulation of leptin in humans. Diabetologia 1997;40:348-51.

25. Mantzoros CS, Prasad AS, Beck F, Grabowski S, Kaplan J, Adair $C$, et al. Zinc may regulate serum leptin concentration in humans. J Am Coll Nutr 1998;17:270-5.

26. Wabitsch $M$, Jensen PB, Blum WF, Christoffersen CT, Englaro $P$, Heinze $E$, et al. Insulin and cortisol promote leptin production in cultured human fat cells. Diabetes 1996;45:1435-8.

27. Papaspyrou-Rao S, Schneider SH, Petersen, RN, Fried SK. Dexamethasone increases leptin expression in humans in vivo. J Clin Endocrinol Metab 1997;82:1635-7.

28. Mantzoros CS, Qu D, Frederich RC, Susulic VS, Lowell BB, Maratos-flier E, et al. Activation of beta (3) adrenergic receptors suppresses leptin expression and mediates a leptin-independent inhibition of food intake in mice. Diabetes 1996;45:909-14.

29. Donahoo WT, Jensen TR, Yost TJ, Eckel RH. Isoproterenol and somatostatin decrease plasma leptin in humans: a novel mechanism regulating leptin secretion. J Clin Endocrinol Metab 1997;82:4139-43.

30. Mantzoros CS, Varvarigou A, Kaklamani VG, Beratis NG, Flier JS. Effect of birth weight and maternal smoking on cord blood leptin concentration of full-term and preterm newborns. J Clin Endocrinol Metab 1997;82:2856-61.

31. Zumbach MS, Boehme MWJ, Wahl P, Stremmel W, Ziegler R, Nawroth PP. Tumor necrosis factor increases serum leptin levels in humans. J Clin Endocrinol Metab 1997:82:4080-2.

32. Sarraf $P$, Frederich RC, Turner EM, Ma G, Jaskowiak NT, Rivet DJ 3rd, et al. Multiple cytokines and acute inflammation raise mouse leptin levels: potential role in inflammatory anorexia. J Exp Med 1997;185(1):171-5.

33. Pérusse L, Collier, Gagnon J, Leon AS, Rao DC, Skinner JS, et al. Acute and chronic effects of exercise on leptin levels in human, J Appl Physiol 1997;83(1):5-10.

34. Sudi K, Jürimäe J, Payerl D, Pihl E, Möller R, Tafeit E, et al. Relationship between subcutaneous fatness and leptin in male athletes. Med Sci Sports Exerc 2001;33(8):1324-9.

35. Engstrom BE, Burman $P$, Holdstock $C$, Karlsson FA. Effect of growth hormone $(\mathrm{GH})$ on ghrelin, leptin, and adiponectin in GH-deficient patients. J Clin Endocrinol Metabol 2003:88:5193-8.

36. Souza MSF, Cardoso AL, Yasbek JRP, Fainthch J. Aerobic endurance, energy expenditure, and serum leptin response in obese, sedentary, prepubertal children and adolescents participating in a short-term treadmill protocol. Nutrition 2004;20:900-4.

37. Umemoto $Y$, Tsuji $K$, Yang FC, Ebihara $Y$, Kaneko $A$, Furukawa $S$, et al. Leptin stimulates the proliferation of murine myelocytic and primitive hematopoietic progenitor cells. Blood 1997;90(9):3438-43.

38. Faggioni $R$, Feingold $K R$, Grunfeld $C$. Leptin regulation of the immune response and the immunodeficiency of malnutrition. FASEB J 2001; 15:2565-71.

39. Hoggard N, Hunter L, Duncan J, Williams LM, Trayhurn $P$, Mercer JG. Leptin and leptin receptor mRNA and protein expression in the murine fetus and placenta. Proc Natl Acad Sci USA 1997:94:11073-8.

40. Winnicki M, Phillips BG, Accurso $V$, van De Borne $P$, Shamsuzzaman A, Patil $K$, et al. Independent association between plasma leptin levels and heart rate in heart transplant recipients. Circulation 2001;104:384-6.

41. Sader S, Nian M, Liu P. Leptin a novel link between obesity, diabetes, cardiovascular risk, and ventricular hypertrophy. Circulation 2003;108:644-6.
42. Thomas $T$, Burguera B. Is leptin the link between fat and bone mass? J Bone Miner Res 2002;17(9):1563-9.

43. Greenway FL, Smith SR. The future of obesity research. Nutrition 2000:16:976-82.

44. Heymsfield SB, Greenberg AS, Fujioka K, Dixon RM, Kushner $R$, Hunt $T$, et al. Recombinant leptin for weight loss in obese and lean adults: a randomized, controlled, dose-escalation trial. JAMA 2000;283(12):1567-8.

45. Steinberg GR, Smith AC, Wormald S, Malenfant P, Collier C, Dyck DJ. Endurance training partially reverses dietaryinduced leptin resistance in rodent skeletal muscle. Am J Physiol Endocrinol Metab 2004:286:E57-63.

46. Kowalska I, Straczkowski M, Gorski J, Kinalska I. The effect of fasting and physical exercise on plasma leptin concentrations in high-fat fed rats. J Physiol Pharmacol 1999;50(2):309-20.

47. Jen CKL, Buison A, Pellizzon M, Ordiz FJR, Ana LS, Brown J. Differential effects of fatty acids and exercise on body weight regulation and metabolism in female Wistar rats. Exp Biol Med 2003;228:843-9.

48. Estadella D, Oyama LM, Dâmaso AR, Ribeiro EB, Nascimento CMD. Effect of palatable hyperlipidic diet on lipid metabolism of sedentary and exercised rats. Nutrition 2004;20:218-24.

49. Leal-Cerro A, Garcia-Luna PP, Astorga R, Parejo J, Peino R, Dieguez $C$, et al. Serum leptin levels in male marathon athletes before and after the Marathon Run. J Clin Endocrinol Metab 1998;83:2376-9.

50. Durstine JL, Thompson RW, Drowatzky KL, Bartoli WP. Leptin and exercise: new directions. Br J Sports Med 2001;35:3-7.

51. Noland RC, Baker JT, Boudreau SR, Kobe RW, Tanner CJ, Hickner RC, et al. Effect of intense training on plasma leptin in male and female swimmers. Med Sci Sports Exerc 2001;33(2):227-31.

52. Kraemer RR, Chu H, Castracane D. Leptin and exercise. Exp Biol Med 2002;227:701-8.

53. Landt M, Lawson GM, Helgeson JM, Davila-Roman VG, Ladenson JH, Jaffe AS, et al. Prolonged exercise decreases serum leptin concentrations. Metabolism 1997;46(10): 1109-12.

54. Racette SB, Coppack SW, Landt M, Klein S. Leptin production during moderate-intensity aerobic exercise. J Clin Endocrinol Metab 1997:82(7):2275-7.

55. Weltman A, Pritzlaff CJ, Wideman L, Considine RV, Fryburg DA, Gutgesell ME, et al. Intensity of acute exercise does not affect serum leptin concentrations in young men. Med Sci Sports Exerc 2000;32(9):1556-61.

56. Olive JL, Miller GD. Differential effects of maximal- and moderate-intensity runs on plasma leptin in healthy trained subjects. Nutrition 2001;17(5):420-2.

57. Zoladz JA, Konturek SJ, Duda K, Majerczak J, Sliwowski Z, Grandys $M$, et al. Effect of moderate incremental exercise, performed in fed and fasted state on cardio-respiratory variables and leptin and ghrelin concentrations in young healthy men. J Physiol Pharmacol 2005;56(1):63-85.

58. Dirlewanger M, Di Vetta V, Giusti V, Schneiter $\mathrm{P}$, Jequier $E$, Tappy L. Effect of moderate physical activity on plasma leptin concentration in humans. Eur J Appl Physiol Occup Physiol 1999;79(4):331-5.

59. Nindl BC, Kraemer WJ, Arciero PJ, Samatallee N, Leone CD, Mayo MF, et al. Leptin concentrations experience a delayed reduction after resistance exercise in men. Med Sci Sports Exerc 2002;34(4):608-13.

60. Essig DA, Alderson NL, Ferguson MA, Bartoli WP, Durstine JL. Delayed effects of exercise on the plasma leptin concentration. Metabolism 2000;49(3):395-9.

61. Keller P, Keller C, Steensberg A, Robinson LE, Pedersen BK. Leptin gene expression and systemic levels in healthy men: effect of exercise, carbohydrate, interleukin-6, and epinephrine. J Appl Physiol 2005;98:1805-12.

62. Ozcelik O, Dogan H, Celik H, Ayar A, Serhatlioglu S, Kelestimur H. Effects of different weight loss protocols on serum leptin levels in obese females. Physiol Res 2005;54(3):271-7. 
63. Pasman WJ, Westerterp-Plantenga MS, Saris WHM. The effect of exercise training on leptin levels in obese males. Am J Physiol 1998;274:E280-6.

64. Reseland JE, Anderssen SA, Solvoll K, Hjermann I, Urdal P, Holme I, et al. Effect of long-term changes in diet and exercise on plasma leptin concentrations. Am J Clin Nutr 2001;73:240-5.

65. Moraes C, Romero CEM, Silva EF, Zanesco A. Serum leptin level in hypertensive middle-aged. Endocrinologist 2005;15:219-21.

66. Gippini A, Mato A, Peino R, Lage M, Dieguez C, Casanueva FF. Effect of resistance exercise (body building) training on serum leptin levels in young men. Implications for relationship between body mass index and serum leptin. $\mathbf{J}$ Endocrinol Invest 1999;22(11):824-8.

67. Ryan AS, Pratley RE, Elahi D, Goldberg AP. Changes in plasma leptin and insulin action with resistive training in postmenopausal women. Int J Obes Relat Metab Disord 2000;24(1)27-32.

68. Zafeiridis A, Smilios I, Considine RV, Tokmakidis SP. Serum leptin responses after acute resistance exercise protocols. J Appl Physiol 2003;94:591-7.

69. Kojima M, Hosoda H, Date Y. Ghrelin is a growth-hormonereleasing acylated peptide from stomach. Nature 1999;402:656-60.

70. Peino R, Baldelli R, Rodriguez-Garcia J, Rodriguez-Segade S, Kojima M, Kangawa K, et al. Ghrelin-induced growth hormone secretion in humans. Eur $J$ Endocrinol 2000;143:R11-4.

71. Date $Y$, Kojima M, Hosoda H, Sawaguchi A, Mondal MS, Suganuma $\mathrm{T}$, et al. Ghrelin, a novel growth hormonereleasing acylated peptide, is synthesized in a distinct endocrine cell type in the gastrointestinal tracts of rats and human. Endocrinology 2000;141:4255-61.

72. Ariyasu H, Takaya K, Tagami T, Ogawa Y, Hosoda K, Akamizu $\mathrm{T}$, et al. Stomach is a major source of circulating ghrelin and feeding state determines plasma ghrelin-like immunoreactivity levels in humans. J Clin Endocrinol Metab 2001:86:4753-8

73. Haqq AM, Farooqi IS, O'Rahilly S, Stadler DD, Rosenfeld RG, Pratt KL, et al. Serum ghrelin levels are inversely correlated with body mass index, age, and insulin concentrations in normal children and are markedly increased in Prader-Willi Syndrome. J Clin Endocrinol Metab 2003;88:174-8.
74. Tschop M, Smiley DL, Heiman ML. Ghrelin induces adiposity in rodents. Nature 2000:407:908-13.

75. Camiña JP. Cell biology of the ghrelin receptor $\mathbf{J}$ Neuroendocrinol 2006;18:65-76.

76. Cummings DE, Clement K, Purnell JQ, Vaisse C, Foster KE, Frayo RS, et al. Elevated plasma ghrelin levels in Prader-Willi Syndrome. Nat Med 2002;8:643-4.

77. Wren AM, Seal LJ, Cohen MA, Brynes AE, Frost GS, Murphy $K G$, et al. Ghrelin enhances appetite and increases food intake in humans. J Clin Endocrinol Metab 2001;86(12):5592-5.

78. English PJ, Ghatei MA, Malik IA, Bloom SR, Wilding JPH. Food fails to suppress ghrelin levels in obese humans. J Clin Endocrinol Metab 2002;87(6):2984-7.

79. Romero CEM, Zanesco A. O papel dos hormônios leptina e grelina na gênese da obesidade. Braz J Nutrition 2006;19:101-4.

80. Dall R, Kanaley J, Hansen TK, Moller N, Christiansen JS, Hosoda $\mathrm{H}$, et al. Plasma ghrelin levels during exercise in healthy subjects and in growth hormone-deficiente patients. Eur J Endocrinol 2002;147:65-70.

81. Morpurgo PS, Resnik M, Agosti F, Cappiello V, Sartorio A, Spada A. Ghrelin secretion in severely obese subjects before and after a 3-week integrated body mass reduction program. J Endocrinol Invest 2003;26:723-7.

82. Foster-Schubert KE, McTiernan A, Frayo RS, Schwartz RS, Rajan KB, Yasui $Y$, et al. Human plasma ghrelin levels increase during a one-year exercise program. J Clin Endocrinol Metab 2005;90:820-5.

83. Ravussin E, Tschöp M, Morales S, Bouchard C, Heiman ML. Plasma ghrelin concentration and energy balance: overfeeding and negative energy balance studies in twins. J Clin Endocrinol Metab 2001;86(9):4547-51.

Endereço para correspondência:

Angelina Zanesco

Departamento de Educação Física

Av. 24A 1515

13506-900 Rio Claro, SP

E-mail: azanesco@rc.unesp.br 